\title{
Adhesion and Structural Changes of PEGylated Lipid Nanocarriers on Silica Surfaces
}

\author{
Philipp Grad ${ }^{1}$, Katarina Edwards 1,*(D) and Víctor Agmo Hernández 2,*(D) \\ 1 Department of Chemistry—Ångström Laboratory, Uppsala University, Box 579, 75123 Uppsala, Sweden; \\ philipp.grad@kemi.uu.se \\ 2 Department of Medicinal Chemistry, Uppsala University, Box 580, 75123 Uppsala, Sweden \\ * Correspondence: katarina.edwards@kemi.uu.se (K.E.); victor.agmo@ilk.uu.se (V.A.H.)
}

check for updates

Citation: Grad, P.; Edwards, K.; Agmo Hernández, V. Adhesion and Structural Changes of PEGylated Lipid Nanocarriers on Silica Surfaces. Physchem 2021, 1, 133-151. https:// doi.org/10.3390/physchem1020009

Academic Editors: Andrea Atrei and Andrea Salis

Received: 29 May 2021

Accepted: 11 July 2021

Published: 15 July 2021

Publisher's Note: MDPI stays neutral with regard to jurisdictional claims in published maps and institutional affiliations.

Copyright: (c) 2021 by the authors. Licensee MDPI, Basel, Switzerland. This article is an open access article distributed under the terms and conditions of the Creative Commons Attribution (CC BY) license (https:// creativecommons.org/licenses/by/ $4.0 /)$.

\begin{abstract}
PEGylated lipid nanoparticles have a continuously expanding range of applications, particularly within pharmaceutical areas. Hereby, it is shown with the help of the Quartz Crystal Microbalance with Dissipation monitoring (QCM-D) and other surface sensitive techniques that, at room temperature, PEGylated liposomes and lipodisks adhere strongly to silica surfaces resulting in the displacement of the hydration layer of silica and the formation of immobilized nanoparticle films. Furthermore, it is shown that drastic changes in the structure of the immobilized films occur if the temperature is increased to $>35^{\circ} \mathrm{C}$. Thus, intact immobilized PEGylated liposomes rupture and spread, even in the gel phase state; immobilized lipodisks undergo complete separation of their components (bilayer forming lipids and PEGylated lipids) resulting in a monolayer of adsorbed PEGylated lipids; and PEGylated supported lipid bilayers release part of the water trapped between the lipid membrane and the surface. It is hypothesized that these changes occur mainly due to the changes in the configuration of PEG chains and a drastic decrease of the affinity of the polymer for water. The observed phenomena can be applied, e.g., for the production of defect-free supported lipid bilayers in the gel or liquid ordered phase states.
\end{abstract}

Keywords: PEGylated liposomes; lipodisks; adsorption; immobilized lipid nanoparticles

\section{Introduction}

Lipid nanocarriers are well established for drug delivery and versatile in their selfassembled structures for many purposes [1]. To increase the colloidal stability of the particles, as well as to prolong their circulation lifetime in vivo, polyethyleneglycol (PEG) grafted lipids or surfactants are commonly added to the preparations [2,3]. This PEGylation strategy has proven useful for a broad range of nanocarriers, including liposomes [4], lipodisks [5], and micelles [6].

Unfortunately, besides the desired increased colloidal stability and prolonged blood survival time, the PEGylation of nanocarriers may lead to some unwanted side effects. For instance, considerable attention has been given to the tendency of PEGylated drug nanocarriers to provoke the production of anti-PEG antibodies, which in turn may result in reduced functionality and toxicity issues [7,8]. Another less recognized and often overlooked problem is that the presence of PEG can result in unwanted interactions between the nanocarriers and certain solid surfaces, such as those composed of silica or the chemically identical quartz. These interactions can lead to biased results from investigations based on spectroscopic analyses with quartz cuvettes and measurements employing Quartz Crystal Microbalance with Dissipation monitoring (QCM-D) in combination with silica sensors [9]. Strategies to avoid or mitigate the PEG-surface interactions are therefore needed. Further, a thorough understanding of the factors affecting the interactions opens up for applications in which the PEG-mediated adsorption is controlled and utilized to obtain surface-immobilized nanocarriers, develop materials for purification/separation, or exploited to produce supported lipid bilayers. 
Despite numerous studies during the last three decades [10-12], the mechanisms behind the interaction of PEG-polymers with hydrophilic surfaces are far from fully understood. In the case of silica it appears that the adsorption of PEG is mainly a result of hydrogen bonding between the ethoxy units of the PEG-polymer and the silanol units on the silica surface, but hydrophobic interactions between PEG-segments and siloxane moieties are believed to play a role as well $[13,14]$. As mentioned above, the interaction between the PEG-polymer and silica can lead to the adsorption of PEG-grafted lipid nanoparticles onto the material [13]. We showed in a recent study that such adsorption can compromise spectroscopic measurements involving the use of quartz cuvettes [15]. However, the interaction between silica surfaces and PEGylated nanocarriers is complex, and several factors, such as $\mathrm{pH}$ and ionic strength, can influence the nanocarriers' affinity for the surface $[13,16]$. Moreover, the PEG-polymer shows an unusual temperature dependent solvation and stretching behavior $[17,18]$, which may translate into an unconventional adsorption behavior.

An additional factor that needs to be taken into account is the composition of the lipid nanocarriers. In line with this, a recent study by Llamas et al. [19] showed that the adsorption of micellar-like aggregates formed by PEG-lipids is strongly influenced by the presence of anionic and zwitterionic surfactants. Taken together, the experimental evidence collected so far indicates that the PEG-mediated particle-surface affinity is determined by a complex interplay of electrostatic and hydrophobic interactions.

In an attempt to shed further light on the phenomena of PEG-mediated adsorption of lipid nanocarriers on hydrophilic surfaces, and learn more about the interaction between PEG and silica, we have in the present study investigated the adsorption behavior on silica of two different types of PEGylated lipid nanocarriers, i.e., liposomes and lipodisks. The latter, which consist of a nanosized lipid bilayer stabilized into a flat circular shape by edge-accumulated PEG-lipids, serve as an interesting comparison to the hollow and less heavily PEGylated liposomes. The interested reader can find more information regarding lipodisks in the study by Zetterberg et al. [20] and the references mentioned therein. By use of a combination of techniques, including multi-parametric surface plasmon resonance (MP-SPR), quartz crystal microbalance with dissipation monitoring (QCM-D) and nanoplasmonic sensing (NPS), we have characterized the binding of the nanocarriers to silica surfaces, as well as investigated their organization and structural transformation upon binding to the solid surface. We have moreover explored how changes in temperature and lipid composition affects the adsorption behavior.

\section{Materials and Methods}

\subsection{Chemicals}

1-palmitoyl-2-oleoyl-sn-glycero-phosphocholin (POPC), 1,2-Distearoyl-sn-glycero-3phosphocholine (DSPC), and 1,2-distearoyl-sn-glycero-3-phosphoethanolamine-N-[methoxy (polyethylene glycol)-2000] ammonium salt (DSPE-PEG2000) were obtained as a kind gift from Lipoid GmbH (Ludwigshafen, Germany). 1,2-distearoyl-sn-glycero-3phosphoethanolamine- $\mathrm{N}-[$ methoxy(polyethylene glycol)-5000] ammonium salt (DSPEPEG5000) and 1,2-distearoyl-sn-glycero-3-phosphoethanolamine-N-[methoxy(polyethylene glycol)-1000] ammonium salt (DSPE-PEG1000) were purchased from Avanti polar lipids (Alabaster, AL, USA). Cholesterol was obtained from VWR.

4-(2-hydroxyethyl)-1-piperazineethanesulfonic acid (HEPES), 2-[(2-Hydroxy-1,1-bis (hydroxymethyl)ethyl)amino]ethanesulfonic acid (TES), sodium phosphate monobasic dihydrate, and sodium chloride were purchased from Sigma Aldrich (Steinheim, Germany). Di-Sodium hydrogen phosphate dihydrate and Chloroform (pro analysis) and methanol (pro analysis) were purchased from Merck KGaG (Darmstadt, Germany). All aqueous solutions were prepared using deionized water $(18.2 \mathrm{M} \Omega \mathrm{cm})$ from a Milli-Q plus system from Millipore (Bedford, MA, USA). Deconex 11 was purchased from Borer Chemie AG (Zuchwil, Switzerland). 


\subsection{Preparation of Liposomes and Lipodisks}

The desired lipids were weighed, or pipetted from stock solutions in chloroform or methanol, and subsequently dissolved in chloroform. A lipid film was obtained by evaporation of the organic solvent, first with a gentle stream of Nitrogen then removing the residual solvent in a vacuum oven from Lab-Line Instruments (Melrose Park, IL, USA) over night.

The lipid film was suspended in either phosphate buffered saline $(10 \mathrm{mM}, 150 \mathrm{mM}$ $\mathrm{NaCl}, \mathrm{pH}=7.4)$ or HEPES buffer $(10 \mathrm{mM}, 150 \mathrm{mM} \mathrm{NaCl}, \mathrm{pH}=7.4)$. The standard protocol for preparation of liposomes and lipodisks consisted of at least 5 and up to 10 freezethawing cycles (freezing with liquid Nitrogen and thawing with a water bath at $60^{\circ} \mathrm{C}$ ) followed by 31 (liposomes) or 21 (disks) times of extrusion through a $100 \mathrm{~nm}$ pore size filter from Whatman plc (Kent, UK).

The lipodisks reported in Section 3.1.1 were prepared by simple hydration of the lipid films in HEPES buffer $(20 \mathrm{mM}, 150 \mathrm{mM} \mathrm{NaCl}, \mathrm{pH}=7.4)$ for $3 \mathrm{~h}$ at $70^{\circ} \mathrm{C}$ with intermittent mixing. The dispersion was kept at $70{ }^{\circ} \mathrm{C}$ and extruded 20 times through a $100 \mathrm{~nm}$ pore size filter from Whatman plc (Kent, UK).

\subsection{QCM-D Characterizations}

The interactions of the different liposomes and lipodisks on various surfaces was monitored with the help of a Quartz Crystal Microbalance with Dissipation monitoring from Q-sense (QCM-D E1, Gothenburg, Sweden). The lipid concentration was set to $1 \mathrm{~g} / \mathrm{L}$ for all experiments unless stated otherwise. Silica (Q-sense, Gothenburg, Sweden) sensors were used. The frequency and dissipation signals were monitored at the fundamental sensor frequency (5 MHz), as well as the 3rd, 5th, 7th, 9th, 11th, and 13th overtones.

The sensors were cleaned before each experiment according to the procedure suggested by the provider. Briefly, the silica sensors were first cleaned in a UV/Ozone chamber from BioForce Nanosciences Inc. (Ames, IA, USA), then immersion of the sensors for 30 min into a $2 \%$ SDS-solution followed by rinsing the sensor with Milli-Q water and drying it with nitrogen and then an additional $30 \mathrm{~min}$ treatment in the UV/ozone chamber.

The sensors were mounted into the QCM-D at a controlled temperature and rinsed with PBS until equilibration was reached. After a stable baseline was obtained the system was loaded with the liposomes or lipodisks with a flow rate of $150 \mu \mathrm{L} / \mathrm{min}$ until a stable signal was obtained. For experiments with temperature cycling, the temperature was programmed in accordance with the settings shown in Table 1.

Table 1. Setting of temperature program.

\begin{tabular}{cc}
\hline Temperature & Time \\
\hline $21{ }^{\circ} \mathrm{C}$ & $10 \mathrm{~min}$ \\
From 21 to $15^{\circ} \mathrm{C}$ & $20 \mathrm{~min}$ \\
$15^{\circ} \mathrm{C}$ & $10 \mathrm{~min}$ \\
From 15 to $60^{\circ} \mathrm{C}$ & $150 \mathrm{~min}$ \\
$60^{\circ} \mathrm{C}$ & $10 \mathrm{~min}$ \\
From 60 to $21^{\circ} \mathrm{C}$ & $130 \mathrm{~min}$ \\
$21^{\circ} \mathrm{C}$ & $10 \mathrm{~min}$ \\
\hline
\end{tabular}

In order to account for the effect of temperature on the background signal, the same program was run on a bare silica sensor on PBS. The data obtained with immobilized liposomes or disks was then corrected by subtraction of the values obtained on bare silica.

The mass density of the immobilized films was calculated from the QCM-D data using the linear equation proposed by Agmo Hernández et al. [21]:

$$
\frac{\Delta f_{n}}{n}=-\frac{m_{d} f_{0}}{t_{q} \rho_{q}}+\frac{\pi \eta_{1}\left(f_{0}\right)^{2}}{\mu_{1}}(n \Delta D)
$$


where $n$ is the overtone number, $f_{0}$ is the fundamental oscillation frequency of the quartz crystal, $t_{\mathrm{q}}$ and $\rho_{\mathrm{q}}$ are the thickness and density of the quartz crystal, $m_{d}$ is the adsorbed mass surface density, $\mu_{1}$ and $\eta_{1}$ are elastic modulus and the viscosity, respectively, of the adsorbed layer. A plot of $\frac{\Delta f}{n}$ vs. $n \Delta D$ at different values of $\mathrm{n}$ should have the y-intercept equal to $-\frac{m_{d} f_{0}}{t_{q} \rho_{q}}$ (i.e., $-m_{\mathrm{d}} /\left(17.7 \mathrm{ng}^{-1} \mathrm{~Hz}^{-1} \mathrm{~cm}^{2}\right)$ for a $5 \mathrm{MHz}$ crystal). Thus the mass density of surface adsorbed layer could be calculated.

\subsection{NPS/QCM-D}

Simultaneous nanoplasmonic sensing and QCM-D experiments were performed using the Acoulyte instrument by Insplorion AB (Gothemburg, Sweden) coupled to a QCM-D QWM 401 window module (Q-Sense, Gothemburg Sweden) connected to the QCM-D E1 instrument. Details concerning the coupled NPS/QCM-D measurements can be found in Ferhan et al. [22]. Liposomes were loaded at a concentration of $0.5 \mathrm{mM}$ and a flow of $150 \mu \mathrm{L} / \mathrm{min}$ until a stable signal was obtained with both techniques. The shift in centroid position in the extinction spectrum was monitored.

\section{5. $M P-S P R$}

The multi-parametric surface plasmon resonance (MP-SPR) measurements reported here correspond to the same experiments previously discussed by Zetterberg et al. [20]. Experimental details can be therefore found there. Briefly, experiments were performed with a SPR Navi 220A (BioNavis, Tampere, Finland) using silica coated gold plasmon surfaces on glass substrates. The surface plasmon resonance was monitored at wavelengths 670 and $785 \mathrm{~nm}$ for a scanning interval between $39^{\circ}$ and $78^{\circ}$. The temperature was held at $21^{\circ} \mathrm{C}$. The lipodisks $(0.25 \mathrm{mM})$ were injected at $50 \mu \mathrm{L} / \mathrm{min}$ to two parallel surface areas of the sensor. After $10 \mathrm{~min}$, the sensors were rinsed with PBS. The bound mass was calculated from the following equation [23]:

$$
m_{d}=\frac{\delta}{S\left(\frac{\mathrm{d} n}{\mathrm{dC}}\right)} \Delta \mathrm{deg}
$$

where $\delta$ is the decay length of the intensity of the evanescent field associated with the SPR excitation (109 and $154 \mathrm{~nm}$ for wavelengths 670 and $785 \mathrm{~nm}$, respectively), $S$ is the bulk sensitivity parameter, $\mathrm{d} n / \mathrm{dC}$ is the refractive index increment $(0.14 \mathrm{~mL} / \mathrm{g})$, as calculated from reported values for lipids and PEG and $\Delta \mathrm{deg}$ is the measurement response. Equation (2) applies only for films much thinner than the decay wavelength $\delta$, a condition that is fulfilled if adsorption of a lipodisk monolayer is assumed.

\section{Results and Discussion}

\subsection{Lipodisks}

\subsubsection{Adsorption on Silica at $21^{\circ} \mathrm{C}$}

Exposure of a silica QCM-D sensor to a $0.25 \mathrm{mM}$ (total lipid) DSPC:DSPE-PEG lipodisk suspension at $21^{\circ} \mathrm{C}$ resulted in the adhesion of lipodisks to the surface (Figure 1) The resulting film could not be removed even after extensive rinsing with buffer, suggesting an irreversible attachment. Noteworthy, repetitions of the experiment resulted in almost exactly the same final surface coverage. This finding is in contrast with previous results observed with active covalent immobilization via amine coupling of lipodisks to NHSactivated gold surfaces, where the degree of surface coverage varied greatly between experiments [21]. Irreversible adhesion of lipodisks was also observed on a silica MP-SPR sensor under the same experimental conditions (Figure 1). 


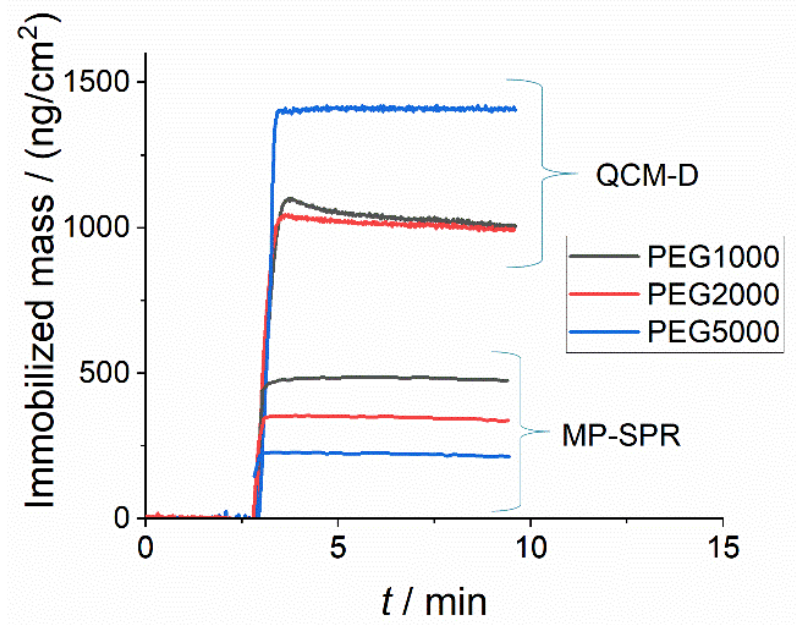

Figure 1. Immobilized lipodisk ( $80 \mathrm{moL} \%$ DSPC and 20 moL\% DSPE-PEG lipids) mass density on silica surfaces as determined using QCM-D (i.e., wet mass) and MP-SPR (dry mass) for several lengths of the PEG-lipid. $T=21^{\circ} \mathrm{C}$.

As observed in Figure 1, the size of the PEG chains affected the weight of the formed film. In the QCM-D experiments, the surface-bound mass was highest for the longest PEGchain (PEG5000). The opposite was observed in the MP-SPR experiments. Given that the main difference between the QCM-D and the MP-SPR is that the former measures also the amount of water in the film while the latter measures only the dry mass, the results indicate that the water content in the immobilized film increases with increasing PEG-length, while the amount of immobilized lipids decreases. These results agree with what is expected given that the steric repulsion between lipodisks will increase with increasing PEG-length, leading to a more sparse distribution of lipodisks on the surface, decreasing thus the lipid content and increasing the contribution of water to the total film mass.

A clearer picture concerning the distribution and orientation of the lipodisks on the surface can be elucidated from the results of the MP-SPR measurements. These suggest that the lipodisk film thickness is in the thin-film range (between 6-10 nm, see Figure S1 in the Supplementary Materials) [24], and is thus much smaller than the diameter of the lipid part of the lipodisks as determined by cryo-TEM (25.4, 26 and $23.1 \mathrm{~nm}$ for disks stabilized by DSPE-PEG1000, DSPE-PEG2000 and DSPE-PEG5000 respectively [20]). This indicates that the lipodisks adsorb parallel to the surface, in contrast with the perpendicular orientation observed in previous reports for disks covalently bound to NHS-activated gold surfaces using amine coupling [21]. This conclusion is further supported by the rather large value of the $\Delta f / \Delta D$ ratio obtained on silica as compared with the value obtained in covalent coupling experiments (for DSPE-PEG2000 stabilized disks on the 3 rd overtone: $20 \times 10^{6} \mathrm{~Hz}$ vs. $4.2 \times 10^{6} \mathrm{~Hz}$, respectively), meaning that a more rigid film is formed on silica, agreeing with lipodisks lying flat on the surface.

The estimated film thickness, together with the large difference between the determined wet and dry masses, and the not negligible value of the dissipation factors measured with the QCM-D, suggests that the film cannot consist only of lipids, but relatively large amounts of water are likely to be trapped in between the lipodisks and the surface, as well as between the lipodisks.

In summary, it can be safely assumed from the results that the lipodisks are oriented parallel to the surface and separated from it by a thin water layer. Figure 2 shows a schematic illustration of the proposed arrangement of lipodisks on the surface. The thickness of the film, is, according to Figure S1 in the Supporting Information, likely to be independent of the PEG-lipid used. On the other hand, and as mentioned above, the data presented in Figure 1 suggest that the space between disk increases with increasing PEG length, due, most likely, to a stronger steric repulsion between immobilized disks. 


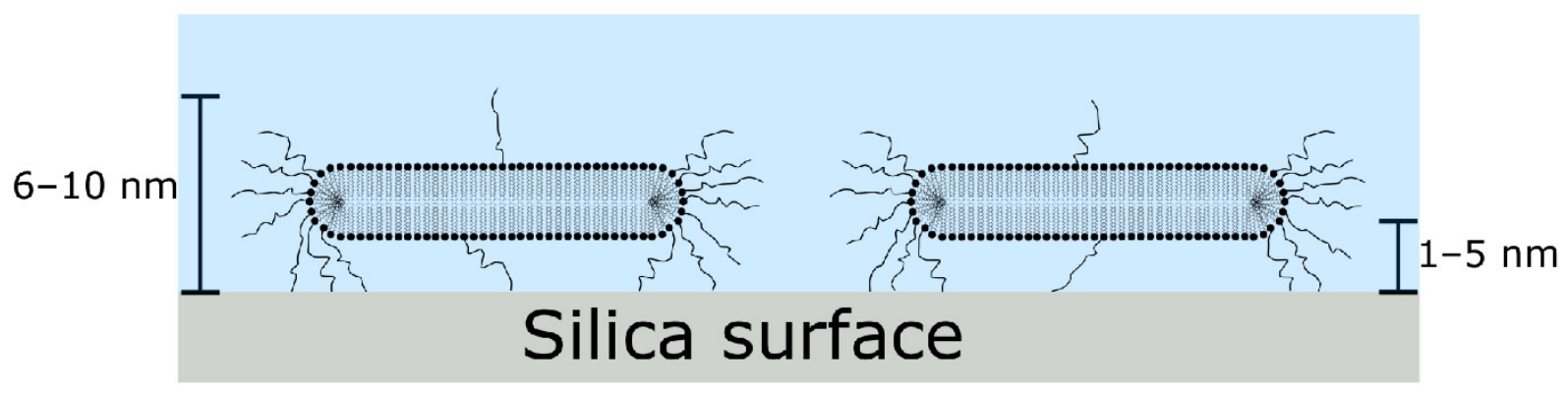

Figure 2. Schematic representation of the proposed arrangement of lipodisks adhered to a silica surface.

The fact that the immobilized film forms spontaneously and is irreversibly attached to the silica substrate indicates a rather high affinity between the lipodisks and the surface. Indeed, experiments performed with low concentrations of lipodisks (down to $1 \mu \mathrm{M}$ total lipid concentration) resulted always in complete coverage of the surface upon long enough (up to $\sim 24 \mathrm{~h}$ ) measurement times (see Figure S2 in the Supplementary Materials), further indicating a very high affinity of the particles for the surface at $21^{\circ} \mathrm{C}$ Previous experiments performed with PEG-micelles [25] have shown a similar high affinity between highly PEGylated nanocarriers and silica.

\subsubsection{Effect of Temperature}

Figure 3 shows the results from experiments probing adhesion of DSPC:DSPE-PEG2000 lipodisks (80:20) to silica at different temperatures. The concentration of lipid was $500 \mu \mathrm{M}$ in all cases. Note that the same batch of lipodisks was used for all the experiments summarized in the figure. The collected data reveals some interesting and somewhat unexpected temperature effects. We will first focus on the behavior in the interval between $21^{\circ} \mathrm{C}$ and $37^{\circ} \mathrm{C}$. It is observed that the initial increase in temperature (up to $27^{\circ} \mathrm{C}$ ) results in a decrease of the recorded mass. This can be interpreted as either a decrease in the surface coverage, or a decrease in the water content of the adsorbed film. Given that the affinity of the lipodisks to the surface is rather high according to the results presented above, the second explanation is more likely. Interestingly, increasing the temperature to $32{ }^{\circ} \mathrm{C}$ resulted in the formation of an immobilized layer that spontaneously seemed to detach from the surface, as illustrated by the fact that the recorded mass suddenly starts decreasing without any action being taken by the experimenter. Rinsing with buffer does not seem to affect this apparent detachment process. This behavior is even more marked at $37^{\circ} \mathrm{C}$, where a rather fast spontaneous adhesion is followed by an apparent spontaneous detachment of the immobilized film. Noteworthy, after this attachment-detachment process the surface remained inactive for lipodisk adhesion, as flowing a fresh lipodisk suspension over the surface resulted only in a negligible amount of disks attaching. These results suggest that at temperatures $\geq 32{ }^{\circ} \mathrm{C}$ the immobilized lipodisks are transformed into a different kind of immobilized structures, with much smaller mass density and the ability to protect the surface against further lipodisk adsorption. It is plausible to assume that these structures are built from PEG-lipids that remain on the surface after the lipodisk detachment. In other words, the results can be explained if it is considered that a few PEGylated lipids are left behind, while the rest of the disk departs from the surface, either as a disk with a lower PEG-content than before the attachment, or as another kind of structure (e.g., a PEGylated vesicle), as illustrated in Figure 4. It has been previously shown that, at this temperature, PEG chains in water tend to acquire an amphiphilic coil-like conformation [26]. Our results suggest that a strong attraction arises when the coil-like conformation becomes dominant resulting in the "pulling out" of PEGylated lipids from lipodisks. This hypothesis will be discussed further in the following sections. 


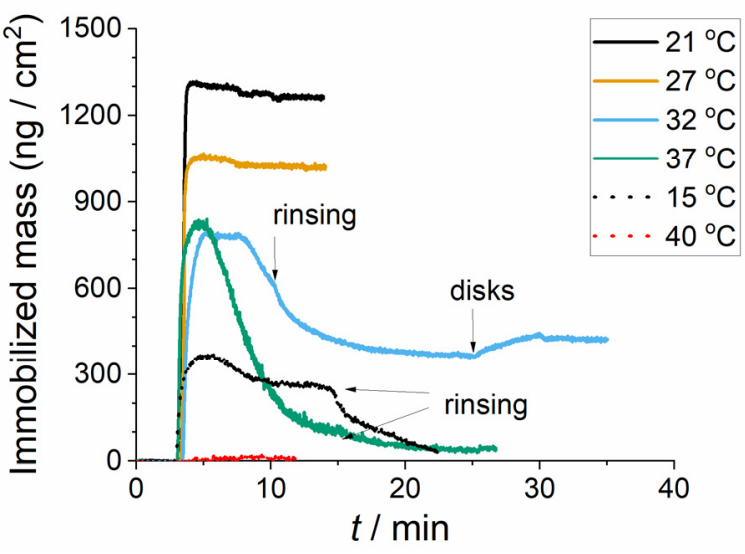

Figure 3. Mass density over time calculated upon the adhesion of DSPC:DSPE-PEG2000 80:20 lipodisks onto QCM-D silica sensors at different temperatures.

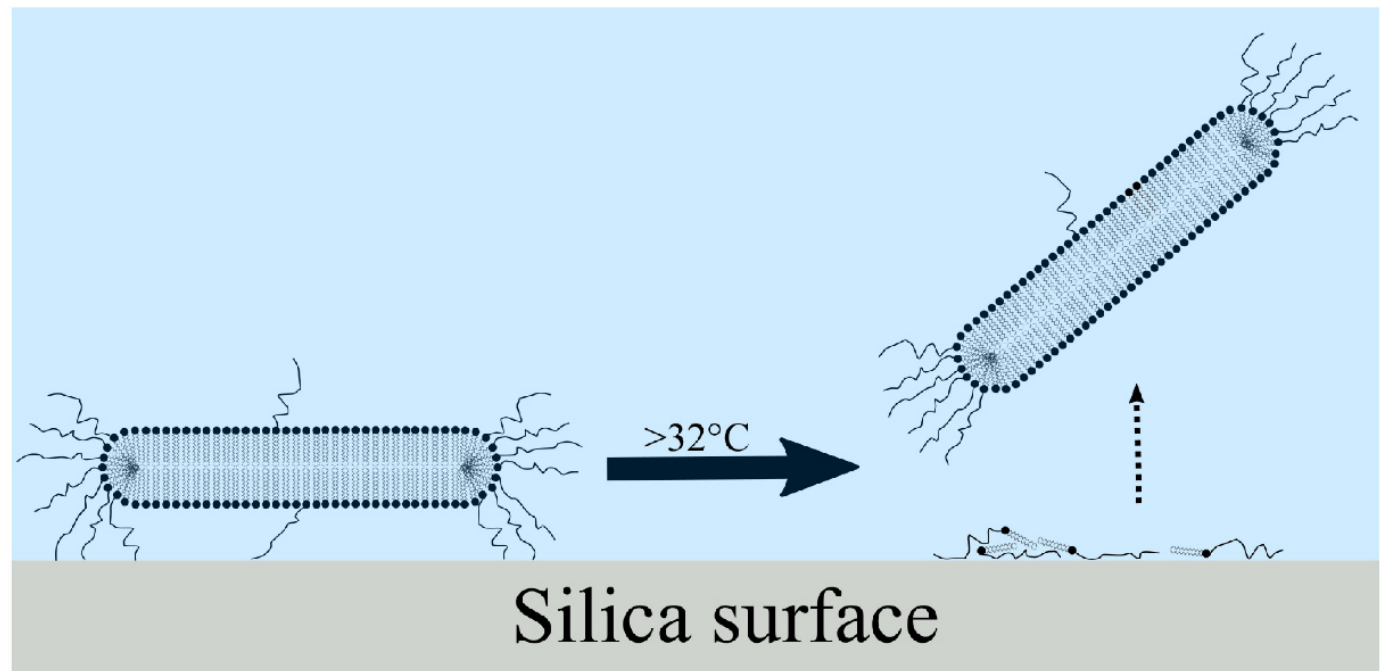

Figure 4. Schematic illustration of the proposed rearrangement of lipodisks on silica surfaces at temperatures higher than $32{ }^{\circ} \mathrm{C}$. PEGylated lipids are pulled out of the lipodisk and remain attached to the surface. The rest of the disk departs from the surface, probably as a disks with lower PEG content than before the attachment.

Interestingly, the experiments performed at $15^{\circ} \mathrm{C}$ and $40{ }^{\circ} \mathrm{C}$ do not follow the trend observed for the rest of the experiments (Figure 3). At $15^{\circ} \mathrm{C}$, a sparsely coated surface is obtained, and the immobilized disks are easily washed away by rinsing, in contrast with what is observed at other temperatures. On the other hand, at $40^{\circ} \mathrm{C}$, no interaction at all was recorded during the experiment. Both observations were reproducible, even when working with different lipodisk batches. It has been previously reported that, at these specific temperatures, the PEG silica interaction results in non-monotonic adsorption isotherms dominated by large concentration ranges in which negative adsorption is observed [26], which can be interpreted as a net repulsion between the polymer and the surface. The fact that at these temperatures no stable adsorption of PEGylated particles can be observed is in line with these observations.

To further investigate the role of temperature, experiments were performed in which the lipodisks were immobilized on the silica sensor at $21^{\circ} \mathrm{C}$ and then subjected to a temperature cycle as described in the methods section. The calculated immobilized mass (after correction for temperature effects on the background signal) is shown in Figure 5. As can be seen, an initial slight decrease in temperature results in a small increase in the immobilized mass. However, upon heating, a rather sudden drop in the measured mass occurs at a temperature $\sim 33.5^{\circ} \mathrm{C}$. At temperatures $\geq 35^{\circ} \mathrm{C}$ no disks are left on the 
surface, although some minor mass changes are observed. Decreasing the temperature does not result in the recovery of the original signal, meaning that no new disks adsorb on the surface and indicating that the latter is coated by a thin film not clearly detected by the instrument.

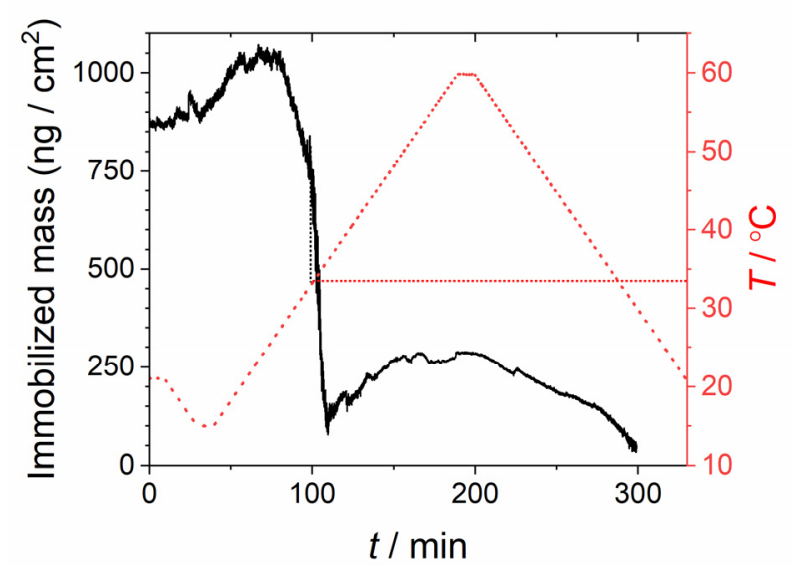

Figure 5. Effect of temperature on the mass of an immobilized film initially consisting of lipodisks at $21^{\circ} \mathrm{C}$. The lipodisks were composed of DSPC:DSPE-PEG2000 with a molar fraction of 80:20.

These observations agree with the experiments performed at different fixed temperatures described above (Figure 3). Taken together, the results indicate that major structural changes of the immobilized material take place when the temperature is increased beyond $\sim 32{ }^{\circ} \mathrm{C}$.

In addition to the investigations performed with lipodisks, QCM-D experiments were carried out with repeated application of DSPE-PEG2000 micelles to a silica sensor (Figure 6). The collected data indicate that the interaction between the micelles and the silica surface is clearly reduced when a second dose of micelles is applied to the sensor. Furthermore, it is not possible to wash of the micelles even after extend times of rinsing the sensor with buffer solution. The reason for this observation is again hypothesized to be that PEG-lipids remain on the sensor after the washing step, and thus reduce the possibility for interactions with subsequently added DSPE-PEG2000 micelles.

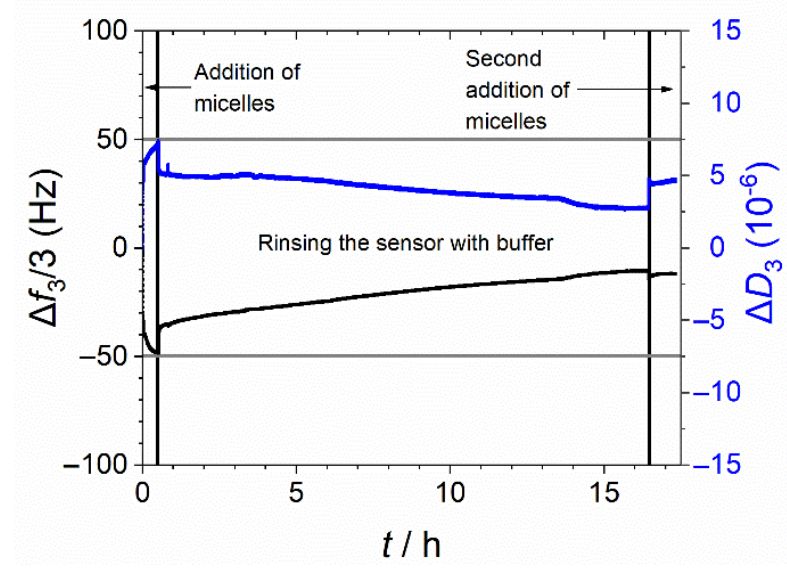

Figure 6. Application of DSPE-PEG2000 micelles on a silica sensor followed by an extended period of rinsing the sensor with buffer solution. Subsequent the micelle solution is inserted again and the interaction between the sensor and the micelles is measured. The vertical black reference lines indicate the start of the rinsing $(t=0.5 \mathrm{~h})$ and the second injection of the micelles solution $(t=16.5 \mathrm{~h})$. The grey horizontal lines indicate the maximum dissipation and minimum frequency shift after the first application of micelles. $\mathrm{T}=21^{\circ} \mathrm{C}$. 


\subsubsection{The Effect of the Hydration Layer on the Interaction of Lipodisks with Silica}

The fact that the release of the lipodisks at $37^{\circ} \mathrm{C}$ results in an immobilized film that is not clearly detected by the QCM-D suggests that the PEG chains are strongly and directly bound to the silica surface. This in turn suggests that the PEG-silica interaction is strong enough to remove the hydration layer on the silica surface. Monovalent counterions at the surface are also likely released. We hypothesized therefore that generating a thicker and more strongly bound hydration layer on silica would hinder the attachment of PEGylated particles to the surface. The effect of the hydration layer on silica and the lipid aggregates was investigated by means of QCM-D experiments in which the HEPES buffer containing $150 \mathrm{mM} \mathrm{NaCl}$ was substituted for HEPES buffer containing $75 \mathrm{mM} \mathrm{CaCl}_{2}$. As can be deduced from the data presented in Figure 7, the buffer change resulted in a markedly different adsorption behavior. In buffer containing $150 \mathrm{mM} \mathrm{NaCl}$ the lipodisks attached to the silica sensor and formed a stable layer that could not be washed off by the buffer (Figure 7a). However, repeating the experiment with a sample dispersed in a $\mathrm{CaCl}_{2}$-containing buffer resulted in frequency and dissipation data indicating a negligible interaction between the lipodisks and the silica sensor (Figure 7b). Similar experiments with lipodisks containing DSPE-PEG5000 instead of DSPE-PEG2000 (Supplementary Materials Figure S3) showed the same trend.
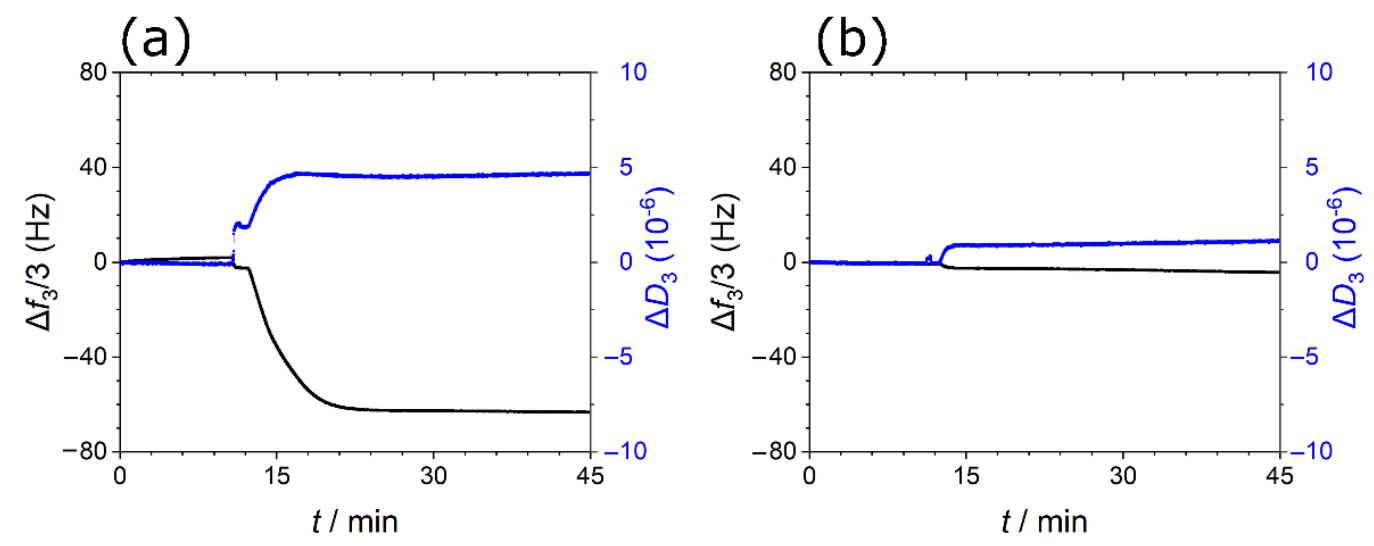

Figure 7. QCM-D graphs of the sample composed of DSPC:DPSE-PEG2000 with molar fractions of 75:25 applied to a silica sensor equilibrated with (a) $10 \mathrm{mM}$ HEPES buffer with $150 \mathrm{mM} \mathrm{NaCl}$ or (b) with $10 \mathrm{mM} \mathrm{HEPES} \mathrm{buffer} \mathrm{with} 75 \mathrm{mM} \mathrm{CaCl}_{2}$. Samples were measured at $25^{\circ} \mathrm{C}$.

The presence of the multivalent calcium ions obviously has an inhibitory effect on the binding of the lipodisks to the silica surface. This suggests that the thicker Stern layers (on both, the lipodisks and, perhaps more importantly, on silica) and stronger hydration forces arising from the presence of multivalent ions [27,28], prevent or decrease, the strength of the interaction between PEG and silica. The stronger hydration repulsive force thereby results in an unfavorable interaction between lipodisks and silica in the presence of calcium ions.

Unlike the well-known calcium triggered fusion process reported with liposomes, neither aggregation nor fusion of the lipodisks was induced by the addition of calcium ions, as confirmed by DLS measurements (Supplementary Materials Figure S4).

\subsection{Liposomes}

Given the unique adhesion behavior of lipodisks onto silica surfaces, we set out to investigate how PEGylation of liposomes affect their interaction with silica substrates. It is well known that non-PEGylated liposomes in the liquid disordered phase state tend to rupture and spread on silica surfaces $[29,30]$ leading to the formation of supported lipid bilayers (SLBs). Liposomes in the gel phase state usually adhere as intact liposomes, while liposomes containing cholesterol have been reported to present mixed behaviors (sometimes adhering as intact liposomes and sometimes spreading), depending on the specific conditions of the experiments [9]. Hereby we investigate the effect of PEGylation 
on the liposome-silica interactions for liposomes in the liquid disordered, gel, and liquid ordered phase states.

\subsubsection{Liposomes in the Liquid Disordered Phase State}

As mentioned above, liposomes formed by a lipid membrane in the liquid disordered phase state usually adhere and spread on silica surfaces. Although the process has mainly been studied using small sonicated liposomes [29], it has been shown that the same applies for larger extruded liposomes based on POPC [9]. Figure 8 indeed shows that extruded POPC liposomes initially adhere on the QCM-D silica sensor and rupture and spread after a few seconds (as evidenced by the drop in frequency and dissipation values). The coating of the surface seems to be incomplete, as adhesion of intact liposomes (as evidenced by changes in both frequency and dissipation factor) is still observed. However, these intact liposomes are rinsed away when clean buffer is introduced. Prolonged rinsing (several hours, results not shown) resulted often in the signal stabilizing at a frequency change of ca $-25 \mathrm{~Hz}$ and small values for the dissipation factor $\left(<<1 \cdot 10^{-6}\right)$, suggesting that a supported lipid bilayer remains on the surface.

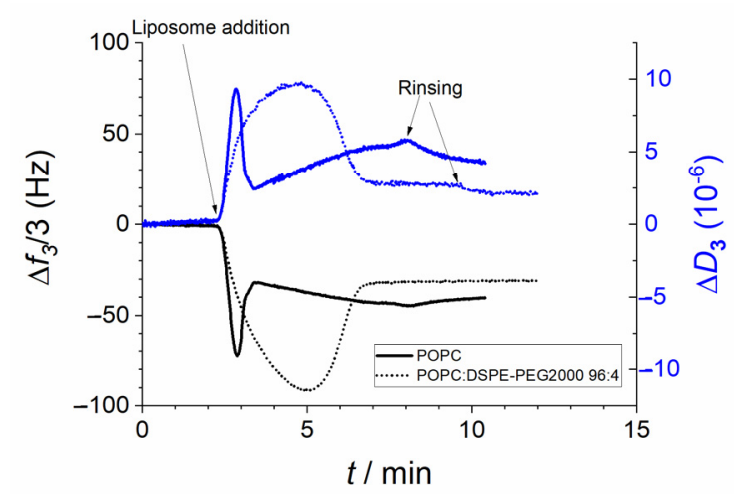

Figure 8. QCM-D signals (3rd overtone) obtained from the adhesion, rupture and spreading of POPC (solid line) and POPC:DSPE-PEG2000 (96:4 moL\%—dotted line) liposomes on a silica surface at $21{ }^{\circ} \mathrm{C}$.

Performing the experiments with liposomes modified with $4 \mathrm{moL} \%$ of PEGylated lipid (Figure 8) resulted in a slower adhesion process, likely due to steric repulsion between adhered and dispersed liposomes. After a few minutes the liposomes eventually ruptured and spread, and no further liposome adhesion was observed. Prolonged rinsing had no effect on the signal obtained. The final values of frequency and dissipation factor changes (-30 Hz and $2 \cdot 10^{-6}$, respectively), suggest a structure heavier and softer than that of a supported POPC bilayer. Applying the same temperature cycle as for the lipodisks (Figure 9), resulted in a decrease in mass as the temperature increased, with a sharp drop at $\sim 35{ }^{\circ} \mathrm{C}$. As for the case of the lipodisks, decreasing the temperature did not result in the recovery of the original mass. The results again suggest that an irreversible change in the immobilized structure takes place at temperatures $>32^{\circ} \mathrm{C}$.

To verify that the effect of temperature is coupled to the presence of PEG lipids and does not arise from the background correction or any other artifact, the same temperature cycle and the same background correction were applied to an SLB formed by pure POPC. As observed in Figure 9, the effect of temperature is in this case the opposite: the mass increases with increasing temperature (likely because more water enters the space between the membrane and the surface), and this increase is reversible (the mass returns to the initial value when the temperature decreases). Thus, it can be concluded that the observed effect of temperature is coupled to the presence of PEG on the immobilized structures. 


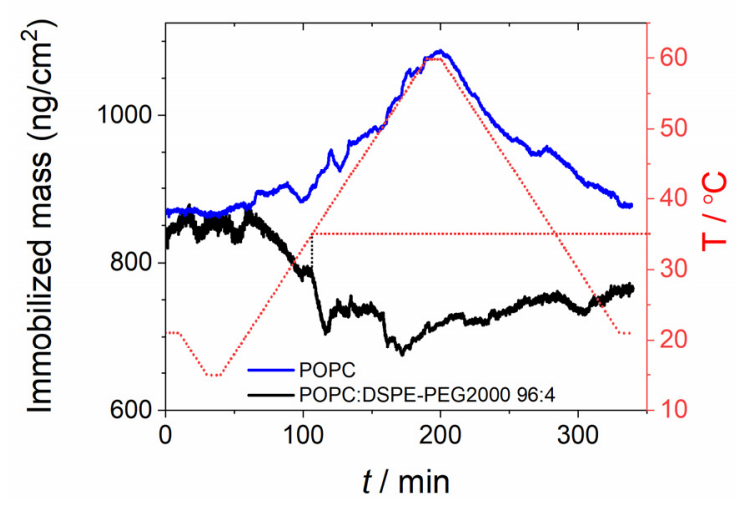

Figure 9. Effect of temperature on the mass (as determined by QCM-D) of supported lipid bilayers formed by POPC (blue line) and by POPC:DSPE-PEG2000 (96:4 moL\%—black line).

\subsubsection{Liposomes in the Gel Phase State}

Both pure DPPC and DPPC:DSPE-PEG2000 (96:4) liposomes in the gel-phase state adhered spontaneously to the silica sensor and remained bound even after extensive rinsing (Figure 10). The main difference between the behavior of the PEGylated and the non-PEGylated liposomes was the larger frequency and dissipation changes observed for the latter, likely because the liposomes can pack more tightly on the surface. Interestingly, for both compositions a peak in the monitored dissipation factor can be observed in the initial stages of the adhesion process. This peak is not reflected in the changes in oscillation frequency. This suggests structural rearrangements of the liposomes occurring at the surface, with the immobilized films becoming more rigid over time. These rearrangements seem to be faster in the case of PEG-containing liposomes.

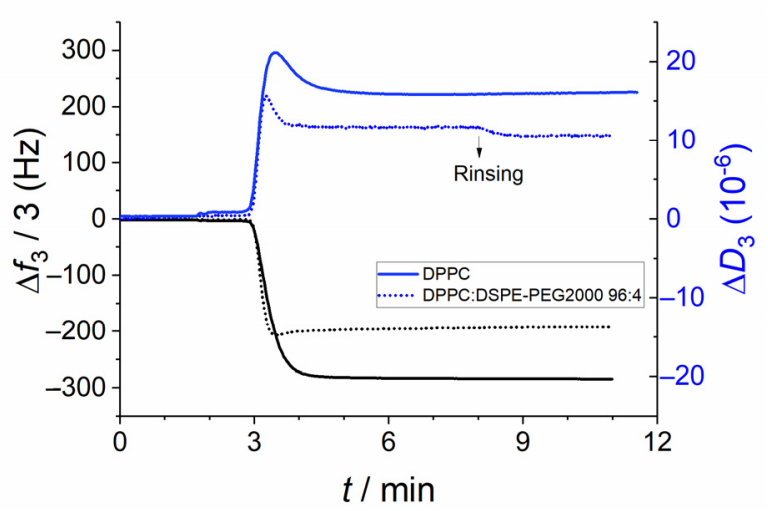

Figure 10. QCM-D signals (3rd overtone) obtained from the adhesion, of DPPC (solid lines) and DPPC:DSPE-PEG2000 (96:4 moL\%—dotted lines) liposomes on a silica surface at $21^{\circ} \mathrm{C}$.

Applying the previously described temperature program to the layer of immobilized liposomes resulted in interesting differences between the PEGylated and non-PEGylated DPPC liposomes (Figure 11). As seen in the figure, in the absence of PEG lipids the liposomes seem to swell as the temperature increases and finally some of them rupture and spread at a temperature corresponding to the main phase transition temperature of the lipids $\left(41^{\circ} \mathrm{C}\right)$. On the other hand, the PEGylated liposome layer decreases in mass as soon as the temperature starts increasing, and there is an abrupt drop in mass, suggesting vesicle rupture and spreading at $35^{\circ} \mathrm{C}$. Note that the signal remains fairly stable after this point. Indeed, repetitions of the experiments resulted in reproducible final values when the liposomes were PEGylated, whereas non-PEGylated DPPC liposomes showed large variations between experiments. This observation can be interpreted as a complete rupture of the liposomes and formation of a supported lipid bilayer in the presence of PEG, while, for non-PEGylated liposomes, whole intact liposomes remain on the surface, even after 
repeated temperature cycles (data not shown). This observation together with the fact that the rupture process occurs at a lower temperature, strongly suggest that PEGylation of the liposomes facilitates their rupture and spreading on the silica surface. This phenomenon has been previously exploited in our laboratory to produce supported DPPC bilayers in the gel-phase state [31,32]. The results from peptide binding experiments performed on these supported structures suggest that the formed bilayers are essentially defect free. To our knowledge, these are the only studies so far that report on the successful formation of supported lipid bilayers in the gel-phase state via vesicle fusion.

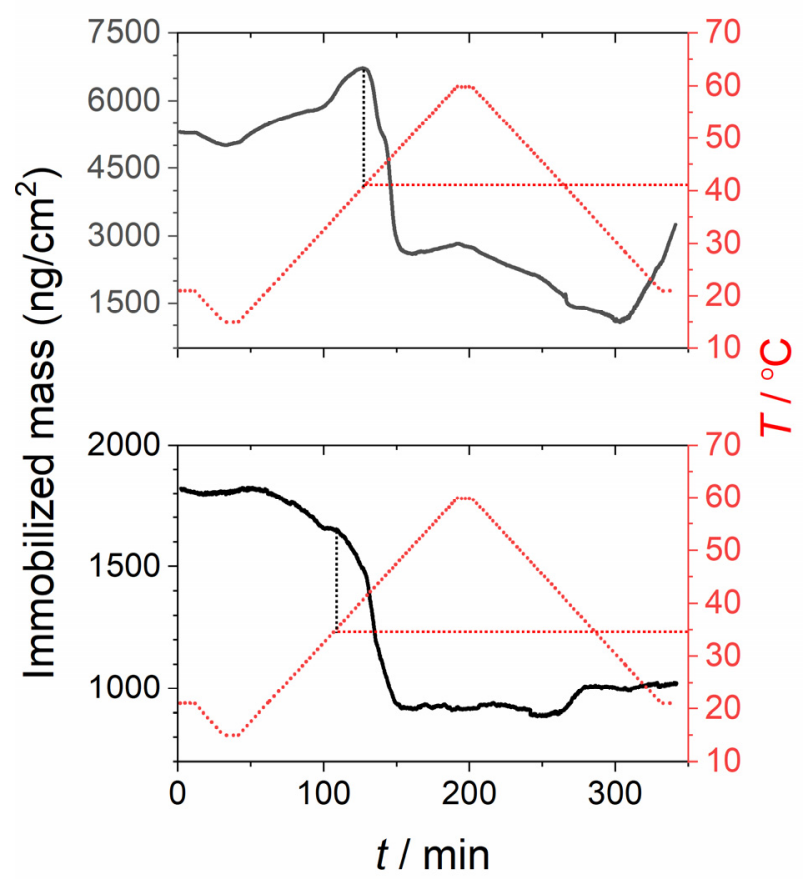

Figure 11. Effect of temperature on the mass (as determined by QCM-D) of immobilized liposome layers formed by DPPC (top) and by DPPC:DSPE-PEG2000 with a molar fraction of 96:4 (bottom).

In order to test whether the same phenomenon would be observed with other lipids in the gel phase state, we repeated the experiments substituting DPPC for DSPC. As for the other liposomes studied, the adhesion of PEGylated liposomes was slightly slower than that of non-PEGylated liposomes (Figure 12). However, in contrast with the results obtained with other compositions, the final shift in oscillation frequency was mostly unaffected suggesting a similar degree of coverage regardless of the presence of PEG-lipids. Another remarkable fact is that after the peak in dissipation, observed right after adhesion, the dissipation signal from PEGylated DSPC liposomes drops steadily, suggesting a continuous "hardening" of the immobilized film.

Interestingly, experiments performed with coupled/simultaneous QCM-D and NPS (Figure 13) showed that the acoustic and the optical signals behave in opposite ways after liposome immobilization. While the wet mass recorded by the QCM-D drops steadily over time, the dry mass (proportional to the shift in centroid position in NPS) continuously increases. This apparent discrepancy can be explained if it is assumed that the liposomes rearrange on the surface in such a way that increasingly more PEG lipids bind to the surface (resulting in an increase in the NPS signal over time) and that in this process water is released from the surface-liposome interspace, resulting in a decrease of the QCM-D signal. Furthermore, the peak observed in the QCM-D signal is not observed in the NPS trace suggesting again a structural rearrangement in which only water is displaced. 


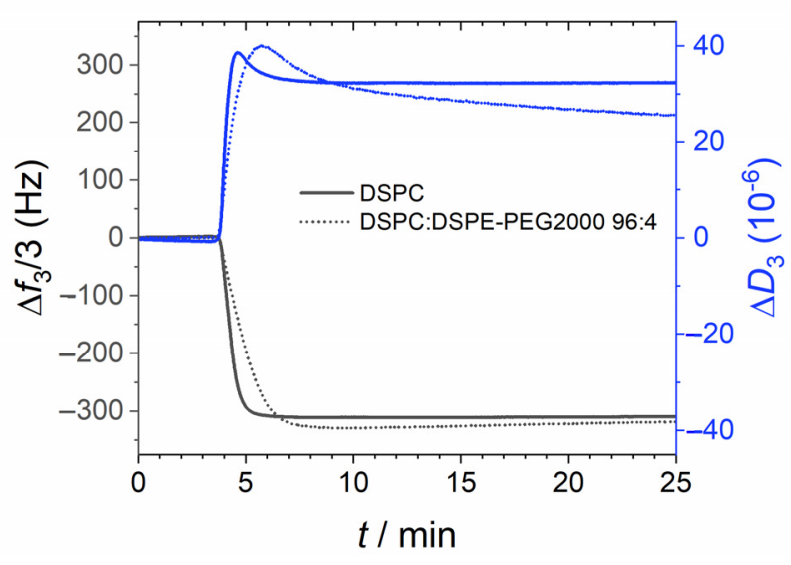

Figure 12. QCM-D signals (3rd overtone) obtained from the adhesion, of DSPC (solid line) and DSPC:DSPE-PEG2000 (96:4 mol\%—dotted line) liposomes on a silica surface at $21^{\circ} \mathrm{C}$.

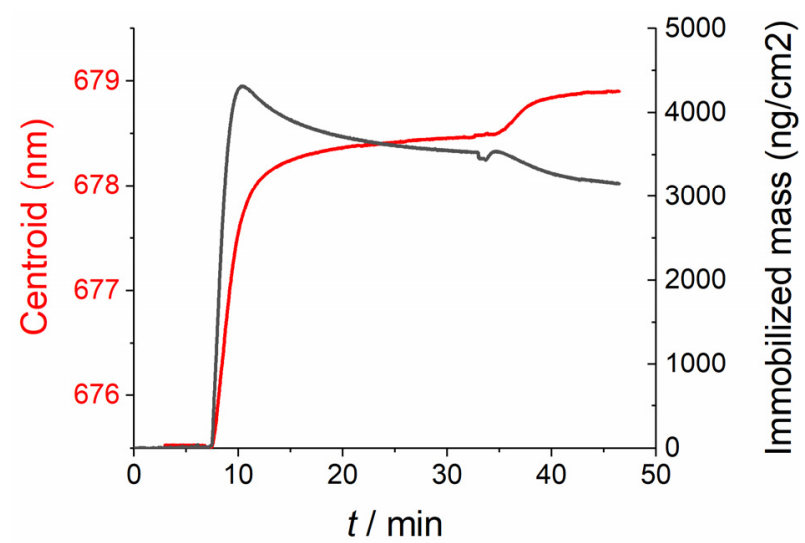

Figure 13. NPS signal and calculated immobilized wet mass (from QCM-D data) obtained from the adhesion, of DSPC:DSPE-PEG2000 (96:4 mol\%) liposomes on a silica surface at $21{ }^{\circ} \mathrm{C}$.

Applying the temperature program to immobilized DSPC-based liposomes resulted in the QCM-D mass densities illustrated in Figure 14. As in the case of DPPC, a sudden drop in the immobilized mass is observed at a temperature corresponding to the phase transition temperature of the lipid $\left(\sim 55^{\circ} \mathrm{C}\right)$ when working with non-PEGylated liposomes. For PEGylated liposomes, the immobilized mass slowly starts decreasing at around $30^{\circ} \mathrm{C}$. This decrease gets continuously faster after $40{ }^{\circ} \mathrm{C}$ and, finally, a sudden drop of the mass is observed at $53{ }^{\circ} \mathrm{C}$. The results suggest that, as in the case of DPPC, the liposomes start rupturing while still in the gel phase. Similarly to DPPC, the signals obtained after liposome rupturing are very stable and reproducible when working the PEGylated liposomes, suggesting the formation of a stable supported lipid bilayer in the gel phase state.

The observed fact that PEGylation enhances liposome rupture and spreading on silica surfaces when the temperature is $>35^{\circ} \mathrm{C}$ is likely to arise from a high affinity of PEG to silica. Although the mechanisms by which liposomes rupture and spread on hydrophilic surfaces are still discussed, it is generally accepted that the process can only occur if the attractive forces between the surface and the membrane are strong enough to deform the liposomes up to the point of rupture [33,34]. Our results suggest that inclusion of PEG does increase the strength of the attractive interactions, and this increase is high enough to induce rupture when the temperature is above $\sim 35^{\circ} \mathrm{C}$. Indeed, it has been previously shown [35] that, at this temperature, the conformation of PEG adsorbed on silica changes (the polymer stretches on the surface) due to a high affinity of the polymer for the surface. As mentioned above, the fact that, even at room temperature, the optical (NPS) signal increases while the QCM-D signal decreases when both are measured simultaneously for 
DSPC-DSPEPEG2000 liposomes is in line with the liposomes flattening on the surface, leading to a curved, stressed edge where rupture can eventually occur (Figure 15).

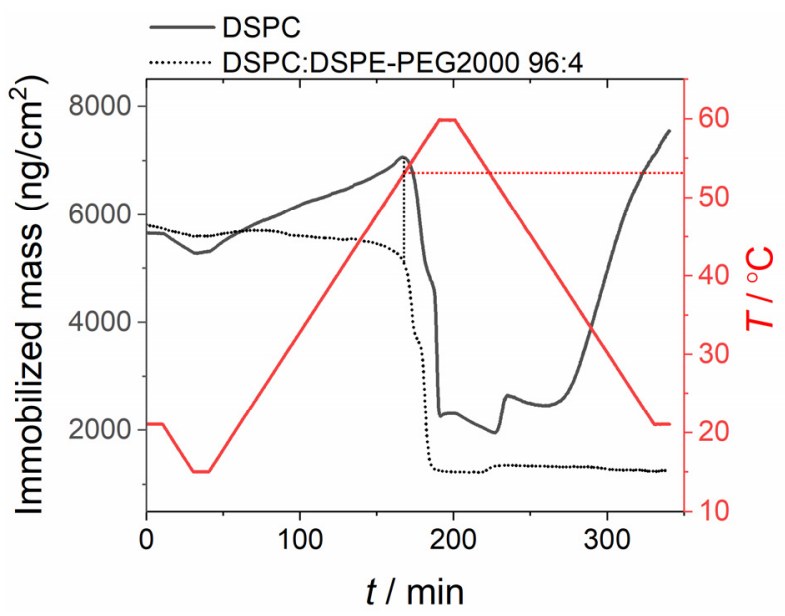

Figure 14. Effect of temperature on the mass (as determined by QCM-D) of immobilized liposome layers formed by DSPC (solid line) and by DSPC:DSPE-PEG2000 (96:4 moL\%-dotted line).

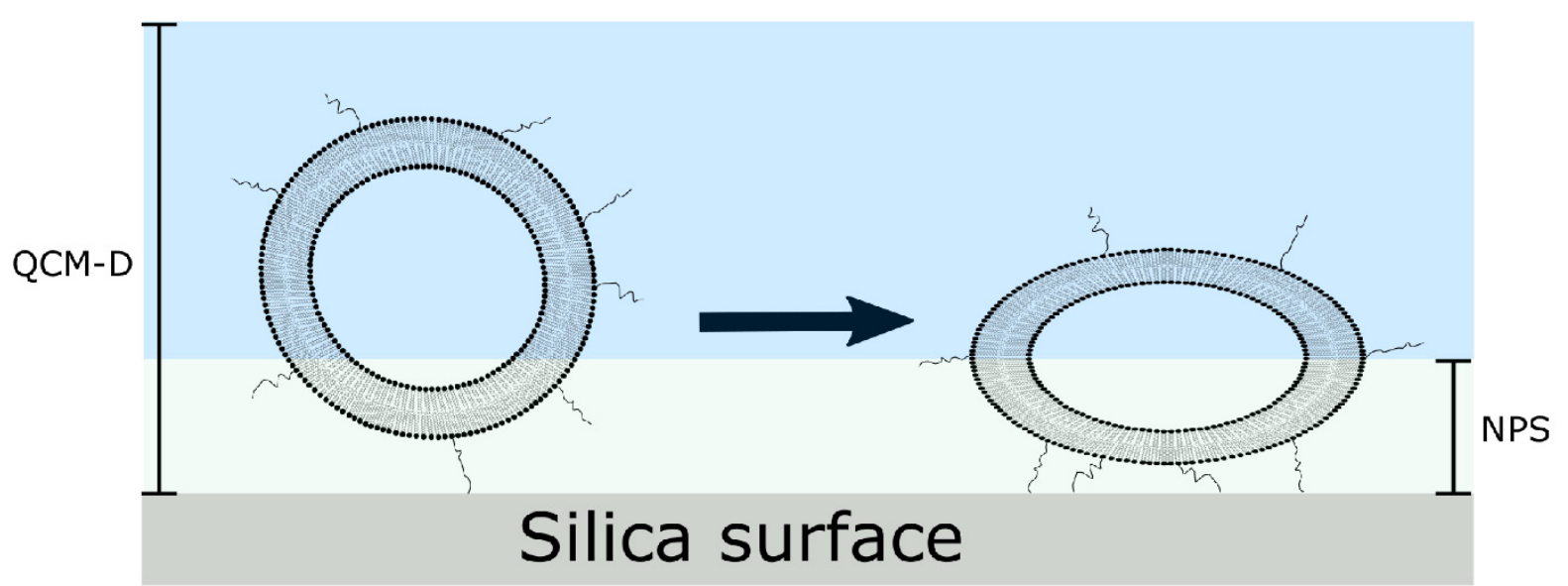

Figure 15. Representation of the proposed rearrangement of PEGylated gel-phase liposomes on silica surfaces. The QCM-D measurement includes the lipids and attached water whereas the NPS measurement only accounts for the lipids.

The question remains concerning why liposomes break while, in the case of lipodisks, the PEGylated lipids seem to be instead detached from the aggregates. A possibility would be that the energy cost of pulling out several PEG-lipids at once from the liposomes is larger than the energy cost of deforming and breaking the membrane. Alternatively, it could be proposed that the former process is kinetically hindered (as several PEG-lipids would need to be detached at once) while the latter can occur much faster. However, this would mean that the release of the bilayer lipids from lipodisks at this temperature would be very slow, which is not the case. A more likely explanation is that, as the amount of PEG in the liposomes is much lower than in the disks, the resulting open bilayer would have enough free space to adsorb on the surface (contrary to the case of lipodisks where most of the surface is covered by PEG-lipids) even if the PEG-lipids are eventually pulled out of the membrane. It can also be speculated that this behavior is linked to the reported transition from amphiphilic helical to hydrophobic coil-like configurations of PEG at $35^{\circ} \mathrm{C}$ [26]. In the case of lipodisks, the local concentration of PEG near the surface is very high and can therefore result in the formation of aggregates when the affinity of the polymer for water decreases. The formation of these aggregates may cause the "pulling-out" of the PEGylated lipids from the lipodisks membrane when the temperature reaches $35^{\circ} \mathrm{C}$. In the case of 
liposomes, the local concentration of PEG is much lower, and it is therefore possible that no such aggregation occurs, leaving thus the PEG-lipids incorporated into the membrane.

\subsubsection{Liposomes in the Liquid Ordered Phase (DSPC:Cholesterol)}

As for liposomes in the liquid disordered (i.e., POPC liposomes) and gel phase (i.e., DPPC and DSPC liposomes) states, inclusion of PEG-lipids resulted in a slower adhesion process when working with liposomes containing cholesterol, i.e., liposomes in the liquid ordered phase state (Figure 16). No liposome rupture was observed even at prolonged times. The peak in dissipation observed for gel phase liposomes is also appreciated in the liquid ordered phase, suggesting that structural rearrangements occur close to the surface. Upon subjecting the immobilized liposome films to the temperature program (Figure 17), it was observed that while non-PEGylated liposomes were barely affected by the temperature change, PEGylated liposomes rupture and spread reproducibly when the temperature reaches ca $33{ }^{\circ} \mathrm{C}$. Given that no phase transition is expected for liposomes of the studied composition, the results suggest that PEG-silica interactions are responsible for this behavior.

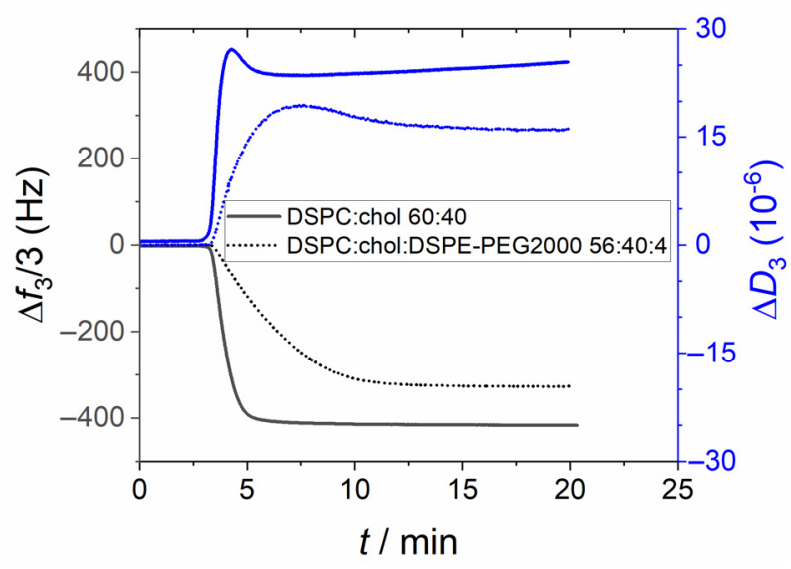

Figure 16. QCM-D signals (3rd overtone) obtained from the adhesion, of DSPC:cholesterol (60:40 moL\%—solid line) and DSPC:cholesterol:DSPE-PEG2000 (56:40:4 moL\%—dotted line) liposomes on a silica surface at $21^{\circ} \mathrm{C}$.

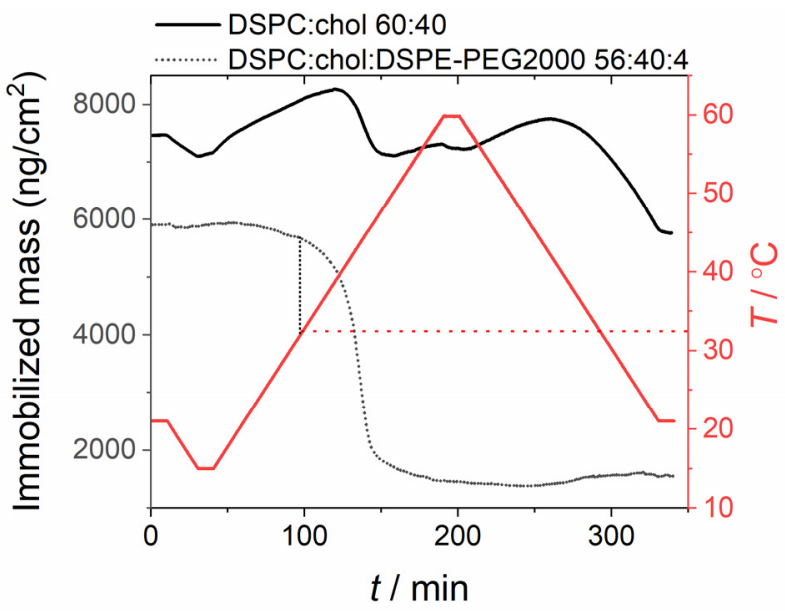

Figure 17. Effect of temperature on the mass (as determined by QCM-D) of immobilized liposome layers formed by DSPC:cholesterol (60:40 moL\%—solid line) and by DSPC:cholesterol:DSPE-PEG2000 (56:40:4 moL\%—dotted line). 


\section{Conclusions}

From the results presented above it is clear that PEGylated liposomes and lipodisks spontaneously attach to silica surfaces at room temperature, forming immobilized layers consisting of either intact liposomes (if using liposomes in the gel or liquid ordered phase states), supported lipid bilayers (in the case of liposomes in the liquid disordered phase state), or intact lipodisks bound parallel to the surface. The characteristics of the formed layers are very reproducible (in terms e.g., of surface coverage and film thickness) for a given composition of the lipid nanoparticles, and the attachment seems to be driven by a high affinity between the PEG chains and the silica surface. Interestingly, the results also show that increasing the temperature leads to irreversible changes in the nature of the supported layers. These changes occur when the temperature reaches $\sim 35^{\circ} \mathrm{C}$, i.e., the same temperature at which previous reports have suggested that the configuration of PEG in solution changes from helical to coil-like and the affinity of the polymer to water decreases drastically [26] while its affinity for silica increases [35]. While in aqueous solutions of PEG the change from helical to coil-like conformations results in a decrease in affinity to silica, likely because a strong repulsion arises between the polymer and the hydration layer on the surface, we show in this work that, for immobilized PEGylated structures, the opposite occurs: i.e., the coil-like conformation gives rise to a strong attraction between the polymer and the surface, resulting in liposome rupture and the "pulling out" of PEGylated lipids from lipodisks. An explanation for this apparent discrepancy is provided by the experiments performed in the presence of calcium. According to the obtained results, when attaching onto silica at room temperature the PEG chains are likely to remove/replace the hydration layer on the surface. Thus, once the temperature is increased to the point where the affinity between PEG and water is low, it is favorable for PEG to adsorb even more strongly onto the already water-depleted silica surface, thus minimizing its interactions with water. This hypothesis is further supported by the fact that immobilizing the particles at room temperature and then increasing the temperature well above $40^{\circ} \mathrm{C}$ does not result in a recovery of the bare surface (meaning that the polymer remains adsorbed on the surface), whereas attempts to perform the immobilization directly at $40{ }^{\circ} \mathrm{C}$ results in no interaction at all (meaning that the polymer is repelled from the surface, as the hydration layer has not been removed).

The described phenomena has already found applications in, e.g., the production of supported lipid bilayers in the gel phase [31,32] (Figure 18). Future applications may include sorting of nanoparticles (e.g., separating them by degree of PEGylation), enrichment of certain proteins or molecules of interest tagged with PEG, and passivation of quartz and other silica-like surfaces to avoid unwanted interactions (as proposed in recent work from our laboratory [15]). 


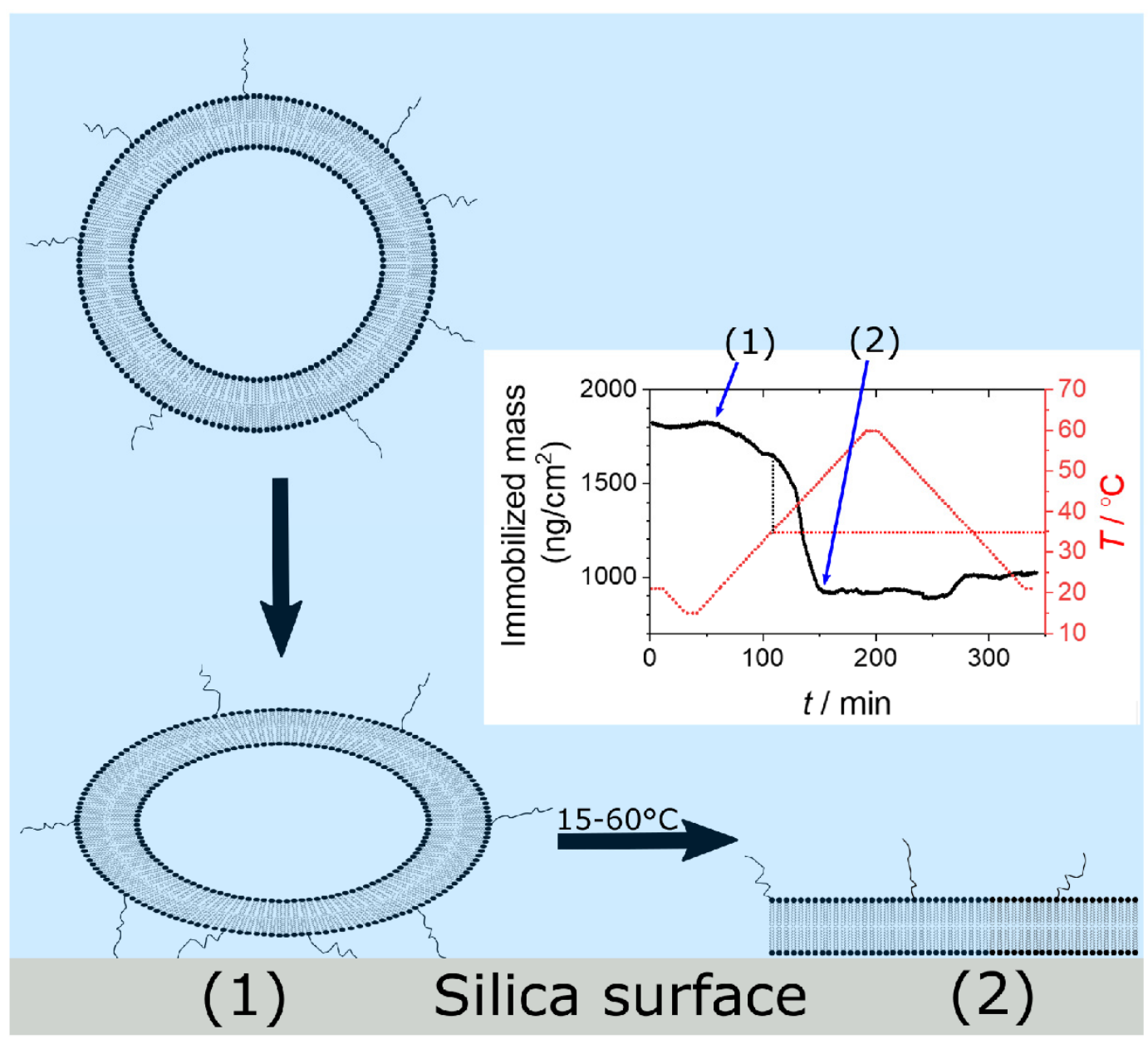

Figure 18. Schematic representation of the formation of supported lipid bilayers out of PEGylated liposomes, in the gel phase state, through temperature mediated rupture processes. The liposomes are attaching and rupturing on the surface (1) and form a supported lipid bilayer (2).

Supplementary Materials: The following are available online at https: / www.mdpi.com/article/ 10.3390/physchem1020009/s1: Figure S1: Calculated thickness of the lipodisks films formed on silica coated MP-SPR sensors, Figure S2: Simultaneously obtained QCM-D calculated mass and centroid position of the NPS signal for $1 \mu \mathrm{M}$ lipodisks adsorbing on a silica sensor, Figure S3: QCM-D graphs of the sample composed of DSPC:DPSE-PEG5000 with molar fractions of 75:25 applied to a silica sensor, Figure S4: Comparative DLS experiments of samples composed of DSPC:DSPE-PEG2000 with molar fractions of 75:25.

Author Contributions: Conceptualization: K.E. and V.A.H.; Methodology: P.G., K.E. and V.A.H.; Validation: P.G. and V.A.H.; Formal analysis: P.G., K.E. and V.A.H.; Investigation: P.G. and V.A.H.; Resources: V.A.H. and K.E.; Writing-original draft preparation: P.G. and V.A.H.; Writing-review and editing: P.G., V.A.H. and K.E.; Supervision: V.A.H. and K.E.; Project administration: K.E.; Funding acquisition: K.E. All authors have read and agreed to the published version of the manuscript.

Funding: This research was funded by the Swedish Research Council (2016-03464) and the Swedish Cancer Society $(17$ 0566, 20 0987).

Institutional Review Board Statement: Not applicable.

Informed Consent Statement: Not applicable.

Data Availability Statement: The data presented in this study is contained within the article or Supplementary Materials.

Acknowledgments: The authors thank Lipoid GmbH for providing some of the lipids used in this work. F. Höök and N. Parveen (Department of Physics, Biological Physics Division, Chalmers Uni- 
versity of Technology, Sweden) are gratefully acknowledged for assistance in the collection and evaluation of MP-SPR data. Insplorion AB (Gothemburg, Sweden) is acknowledged for providing access to the Acoulyte instrument and for assistance with the interpretation of the NPS data. Valuable assistance from Xinchen Zhang and Dimitrios Georgouvelas with the experimental work is greatly appreciated.

Conflicts of Interest: The authors declare no conflict of interest.

\section{References}

1. Drescher, S.; van Hoogevest, P. The Phospholipid Research Center: Current Research in Phospholipids and Their Use in Drug Delivery. Pharmaceutics 2020, 12, 1235. [CrossRef]

2. Needham, D.; McIntosh, T.J.; Lasic, D.D. Repulsive interactions and mechanical stability of polymer-grafted lipid membranes. BBA Biomembr. 1992, 1108, 40-48. [CrossRef]

3. Woodle, M.C.; Lasic, D.D. Sterically stabilized liposomes. BBA-Rev. Biomembr. 1992, 1113, 171-199. [CrossRef]

4. Blume, G.; Cevc, G. Liposomes for the sustained drug release in vivo. BBA Biomembr. 1990, 1029, 91-97. [CrossRef]

5. Ahlgren, S.; Reijmar, K.; Edwards, K. Targeting lipodisks enable selective delivery of anticancer peptides to tumor cells. Nanomedicine 2017, 13, 2325-2328. [CrossRef] [PubMed]

6. Kanwaldeep, K.G.; Kaddoumi, A.; Nazzal, S. PEG-lipid micelles as drug carriers: Physiochemical attributes, formulation principles and biological implication. J. Drug Target. 2015, 23, 222-231.

7. Johan, J.F.V.; Carpenter, J.F.; Anchordoquy, T.J.; Schellekens, H. Potential induction of anti-PEG antibodies and complement activation toward PEGylated therapeutics. Drug Discov. Today 2014, 19, 1945-1952.

8. Anchordoquy, T.J.; Simberg, D. Watching the gorilla and questioning delivery dogma. J. Control. Release 2017, 262, 87-90. [CrossRef] [PubMed]

9. Eriksson, E.K.; Agmo Hernández, V. Choice of cuvette material can influence spectroscopic leakage and permeability experiments with liposomes. Chem. Phys. Lipids 2018, 215, 63-70. [CrossRef] [PubMed]

10. Mathur, S.; Moudgil, B.M. Adsorption Mechanism(s) of Poly(Ethylene Oxide) on Oxide Surfaces. J. Colloid Interface Sci. 1997, 196, 92-98. [CrossRef]

11. Wind, B.; Killmann, E. Adsorption of polyethylene oxide on surface modified silica-Stability of bare and covered particles in suspension. Colloid Polym. Sci. 1998, 276, 903-912. [CrossRef]

12. Char, K.; Alice, G.; Curtis, F. Fluorescence studies of polymer adsorption. 1. Rearrangement and displacement of pyreneterminated polyethylene glycol on colloidal silica particles. Langmuir 1988, 4, 989-998. [CrossRef]

13. Chang, D.P.; Jankunec, M.; Barauskas, J.; Tiberg, F.; Nylander, T. Adsorption of Lipid Liquid Crystalline Nanoparticles on Cationic, Hydrophilic, and Hydrophobic Surfaces. ACS Appl. Mater. Interface 2012, 4, 2643-2651. [CrossRef] [PubMed]

14. Rubio, J.; Kitchener, J.A. The mechanism of adsorption of poly(ethylene oxide) flocculant on silica. J. Colloid Interface Sci. 1976, 57, 132-142. [CrossRef]

15. Grad, P.; Agmo Hernández, V.; Edwards, K. Avoiding artifacts in liposome leakage measurements via cuvette- and liposomesurface modifications. J. Lipid Res. accepted.

16. Iruthayaraj, J.; Poptoshev, E.; Vareikis, A.; Makuška, R.; van der Wal, A.; Claesson, P.M. Adsorption of Low Charge Density Polyelectrolyte Containing Poly(ethylene oxide) Side Chains on Silica: Effects of Ionic Strength and pH. Macromolecules 2005, 38, 6152-6160. [CrossRef]

17. Liese, S.; Gensler, M.; Krysiak, S.; Schwarzl, R.; Achazi, A.; Paulus, B.; Hugel, T.; Rabe, J.P.; Netz, R.R. Hydration Effects Turn a Highly Stretched Polymer from an Entropic into an Energetic Spring. ACS Nano 2017, 11, 702-712. [CrossRef]

18. Kolberg, A.; Wenzel, C.; Hackenstrass, K.; Schwarzl, R.; Rüttiger, C.; Hugel, T.; Gallei, M.; Netz, R.R.; Balzer, B.N. Opposing Temperature Dependence of the Stretching Response of Single PEG and PNiPAM Polymers. J. Am. Chem. Soc. 2019, 141, 11603-11613. [CrossRef]

19. Llamas, S.; Guzmán, E.; Ortega, F.; Rubio, R.G. Adsorption of Mixtures of a Pegylated Lipid with Anionic and Zwitterionic Surfactants at Solid/Liquid. Colloids Interfaces 2020, 4, 47. [CrossRef]

20. Zetterberg, M.M.; Ahlgren, S.; Hernández, V.A.; Parveen, N.; Edwards, K. Optimization of lipodisk properties by modification of the extent and density of the PEG corona. J. Colloid Interface Sci. 2016, 484, 86-96. [CrossRef]

21. Agmo Hernández, V.; Reijmar, K.; Edwards, K. Label-Free Characterization of Peptide-Lipid Interactions Using Immobilized Lipodisks. Anal. Chem. 2013, 85, 7377-7384. [CrossRef]

22. Ferhan, A.R.; Jackman, J.A.; Cho, N.-J. Integration of Quartz Crystal Microbalance-Dissipation and Reflection-Mode Localized Surface Plasmon Resonance Sensors for Biomacromolecular Interaction Analysis. Anal. Chem. 2016, 88, 12524-12531. [CrossRef]

23. Jung, L.S.; Campbell, C.T.; Chinowsky, T.M.; Mar, M.N.; Yee, S.S. Quantitative interpretation of the response of surface plasmon resonance sensors to adsorbed films. Langmuir 1998, 14, 5636-5648. [CrossRef]

24. Rupert, D.L.M.; Shelke, G.V.; Emilsson, G.; Claudio, V.; Block, S.; Lässer, C.; Dahlin, A.; Lötvall, J.O.; Bally, M.; Zhdanov, V.P.; et al. Dual-Wavelength Surface Plasmon Resonance for Determining the Size and Concentration of Sub-Populations of Extracellular Vesicles. Anal. Chem. 2016, 88, 9980-9988. [CrossRef] [PubMed] 
25. Saigal, T.; Riley, J.K.; Lynn Golas, P.; Bodvik, R.; Claesson, P.M.; Matyjaszewski, K.; Tilton, R.D. Poly(Ethylene Oxide) Star Polymer Adsorption at the Silica/Aqueous Interface and Displacement by Linear Poly(Ethylene Oxide). Langmuir 2013, 29, $3999-4007$. [CrossRef] [PubMed]

26. Nawal, D.; Sylvère, S.; Yves, G.; René, O.; Mireille, P. Polyethylene Glycol Adsorption on Silica: From Bulk Phase Behavior to Surface Phase Diagram. Langmuir 2007, 23, 6631-6637.

27. Pashley, R.M.; Israelachvili, J.N. Dlvo and hydration forces between mica surfaces in Mg2+, Ca2+, Sr2+, and Ba2+ chloride solutions. J. Colloid Interface Sci. 1984, 97, 446-455. [CrossRef]

28. Pashley, R.M.; Quirk, J.P. The effect of cation valency on DLVO and hydration forces between macroscopic sheets of muscovite mica in relation to clay swelling. Colloid Surf. 1984, 9, 1-17. [CrossRef]

29. Keller, C.A.; Kasemo, B. Surface specific kinetics of lipid vesicle adsorption measured with a quartz crystal microbalance. Biophys. J. 1998, 75, 1397-1402. [CrossRef]

30. Keller, C.A.; Glasmastar, K.; Zhdanov, V.P.; Kasemo, B. Formation of supported membranes from vesicles. Phys. Rev. Lett. 2000, 84, 5443-5446. [CrossRef]

31. Reijmar, K.; Edwards, K.; Andersson, K.; Agmo Hernandez, V. Characterizing and controlling the loading and release of cationic amphiphilic peptides onto and from PEG-stabilized lipodisks. Langmuir ACS J. Surf. Colloids 2016, 32, 12091-12099. [CrossRef]

32. Lundsten, S.; Agmo Hernandez, V.; Gedda, L.; Saren, T.; Brown, C.J.; Lane, D.P.; Edwards, K.; Nestor, M. Tumor-Targeted Delivery of the p53-Activating Peptide VIP116 with PEG-Stabilized Lipodisks. Nanomaterials 2020, 10, 783. [CrossRef]

33. Seifert, U.; Lipowsky, R. Adhesion of Vesicles. Phys. Rev. A 1990, 42, 4768-4771. [CrossRef]

34. Richter, R.P.; Bérat, R.; Brisson, A.R. Formation of Solid-Supported Lipid Bilayers: An Integrated View. Langmuir 2006, 22, 3497-3505. [CrossRef]

35. Wiśniewska, M.; Szewczuk-Karpisz, K.; Ostolska, I. Temperature effect on the adsorption equilibrium at the silica-polyethylene glycol solution interface. Fluid Phase Equilibr. 2013, 360, 10-15. [CrossRef] 